\title{
Teleultrasound: Historical Perspective and Clinical Application
}

\author{
Adilson Cunha Ferreira, ${ }^{1,2,3}$ Edward O'Mahony, ${ }^{1}$ Antonio Hélio Oliani, ${ }^{2}$ \\ Edward Araujo Júnior, ${ }^{4}$ and Fabricio da Silva Costa ${ }^{1,3}$ \\ ${ }^{1}$ Department of Obstetrics and Gynaecology and Department of Perinatal Medicine, Pregnancy Research Centre, \\ Royal Women's Hospital, University of Melbourne, Melbourne, VIC 3207, Australia \\ ${ }^{2}$ School of Medicine, University of São José do Rio Preto, São José do Rio Preto, SP, Brazil \\ ${ }^{3}$ Monash Ultrasound for Women, Melbourne, VIC, Australia \\ ${ }^{4}$ Department of Obstetrics, Paulista School of Medicine-Federal University of São Paulo (EPM-UNIFESP), \\ 05303-000 São Paulo, SP, Brazil
}

Correspondence should be addressed to Adilson Cunha Ferreira; adilsonteleultrassonografia@gmail.com

Received 12 September 2014; Accepted 8 February 2015

Academic Editor: Sotiris A. Pavlopoulos

Copyright (C) 2015 Adilson Cunha Ferreira et al. This is an open access article distributed under the Creative Commons Attribution License, which permits unrestricted use, distribution, and reproduction in any medium, provided the original work is properly cited.

The health care of patients in rural or isolated areas is challenged by the scarcity of local resources, limited patient access to doctors and hospitals, and the lack of specialized professionals. This has led to a new concept in telemedicine: teleultrasonography (or teleultrasound), which permits ultrasonographic diagnoses to be performed remotely. Telemedicine and teleultrasonography are effective in providing diagnostic imaging services to these populations and reduce health care costs by decreasing the number and duration of hospitalizations and reducing unnecessary surgical procedures. This is a narrative review to present the potential clinical applications of teleultrasonography in clinical practice. The results indicate that although barriers persist for implementing teleultrasonography in a more universal and routine way, advances in telecommunications, Internet bandwidth, and the high resolution currently available for portable ultrasonography suggest teleultrasonography applications will continue to expand. Teleultrasound appears to be a valuable addition to remote medical care for isolated populations with limited access to tertiary healthcare facilities and also a useful tool for education and training.

\section{Introduction}

Under the WHO definition of telemedicine, "the delivery of health care services, where distance is a critical factor," it is intended for the exchange of valid information for the diagnosis, prevention, and treatment of disease and for the continuing education of health service providers, as well as for research and evaluation purposes [1]. Ultrasonography is very useful diagnostic tool because it is a noninvasive, generally nonexpensive, and highly portable method that does not use ionizing radiation [2]. However, generating and interpreting ultrasound images are highly operator-dependent. As a result, performance and interpretation of these examinations have traditionally been limited to medical specialists $[3,4]$. Although some remote areas have access to basic primary care services, including X-rays and ultrasound, they frequently lack specialized radiologists and ultrasonographers [5]. This lack of experienced doctors or qualified technicians has led to a new concept in telemedicine: teleultrasonography (or teleultrasound), which permits ultrasonographic examinations to be performed remotely in a synchronous (real-time) or asynchronous fashion. Over the past decade, research on teleultrasonography has evolved incrementally, especially with regard to technology, which is why most of the work has been published in technical engineering and medical informatics journals. However, few studies have been published on training doctors and technicians from remote or isolated areas.

This paper presents a narrative review of the potential clinical applications of teleultrasound in clinical practice. 


\section{Historical Perspectives}

The committee formed by the collaboration between ACR, American College of Radiology, and NEMA, National Electrical Manufacturers Association in 1983, aimed to solve the problem of the Babel of files formats, images, and information generated by different manufacturers and equipment and to define the parameters for the transmission of distance medical images in an asynchronous way. From this point onwards, several studies were carried out in order to prove the diagnostic accuracy of this type of transmission, which is well established today [6-8].

Among the different areas of teleradiology, teleultrasonography is potentially the safest and least expensive area because it does not utilize ionizing radiation and is affordable $[3,4]$. Investigators in the United States, the United Kingdom, Canada, and Australia were the pioneers in this research and in carrying out pilot studies [5-9]. The majority of these projects were based on the use of hardware and software packages which required high speed broadband connections and were associated with a high cost of implementation [5].

\section{Transmission Modes}

The asynchronous method is noninteractive. In this mode, data are collected, stored, and then forwarded to a sonologist for interpretation. This system can capture and store images as well as audio and text. This mode eliminates the need for the doctor and patient to be present at the same time in the same place and is widely used in countries where technicians instead of doctors perform ultrasound examinations $[7,10]$. It is also widely used in university hospitals where trainees perform the examination and save the images, which are then evaluated together with the supervising physician $[7,11]$. This type of service increases population access to services that are not locally available and is currently the most widespread mode of using teleultrasonography $[11,12]$. In the synchronous mode, data transmission takes place in real time, that is, while the examination is being performed. This mode enables more experienced doctors to remotely supervise any person who is operating the ultrasound equipment, including technicians, medical residents, and personnel in training [12]. This system reduces the need for face-to-face consultations and allows for earlier detection of problems, which may further reduce the costs associated with the delocalization of specialized personnel. In the medical field, this modality involves a real-time interaction between the patient, the person operating the ultrasound equipment, and the interpreting specialist who will report the final diagnosis. This mode is not only possible but is well accepted by the physicians and patients involved. With the widespread use of the Internet across the world and increased communication via fixed or wireless broadband Internet access, the synchronous mode should become an important tool for clinical practices and educational purposes [13-15].

\section{Research Categories and Themes}

Research on teleultrasonography can be divided into studies that use synchronous (real-time) (Figure 1) and asynchronous
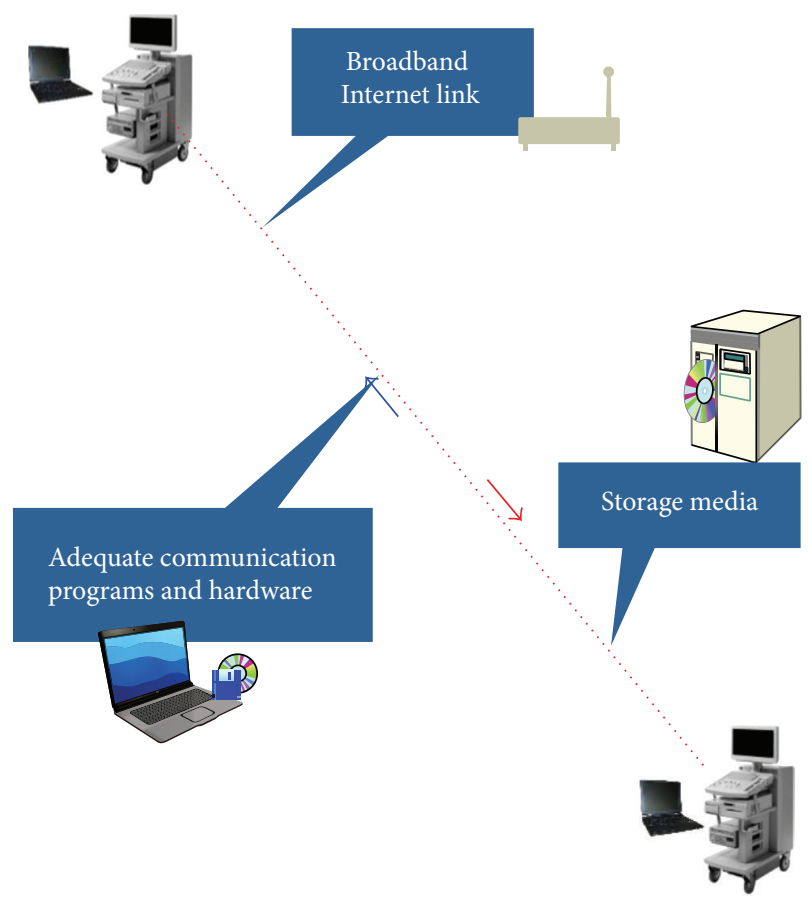

FIGURE 1: Illustrative description of the system utilized to real-time transmission of video clips and images between different centers.

(store-and-forward) transmission. Within these two categories of research, there are three recurring themes that are relevant to the successful implementation of a teleultrasonography system: (a) the quality of the transmitted images; (b) clinical applications; (c) the nontechnical and technical barriers to implementation [4].

Opinions in the literature are divided with regard to the transmission system involved in teleultrasonography. Some authors consider that good image quality can only be obtained with the use of asynchronous transmission, which, in addition to good diagnostic accuracy, allows for the training and professional supervision to produce a satisfactory level of clinical competence $[6-8,10,12]$. Other studies have sought to demonstrate the accuracy of the teleultrasonography performed in real time between a tertiary center and a remote area. The authors argue that the image quality was not very clear when teleultrasonography first started but that current telecommunication and image compression technologies have made high-quality synchronous and asynchronous transmissions feasible $[5,16]$. Other authors argue in favour of real-time transmissions because the asynchronous mode only allows images and videos to be stored for future analysis and their interpretation may be incomplete or diagnostically inaccurate if some important information is missing and cannot be recovered [17]. However, the increasingly routine use of real-time transmission is still associated with high costs, and various studies have proposed alternatives to reduce these costs $[5,18-20]$.

\section{Image Quality}

Studies focused on the quality of transmitted images have attempted to determine the minimum bandwidth required, 

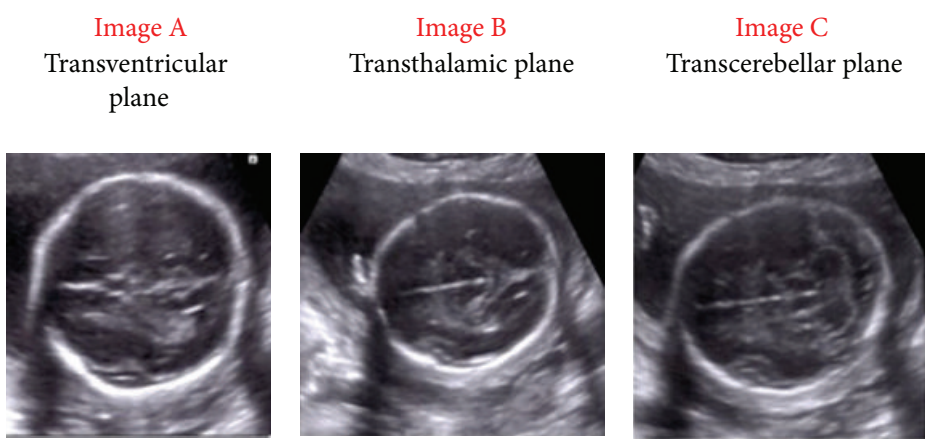

(a)
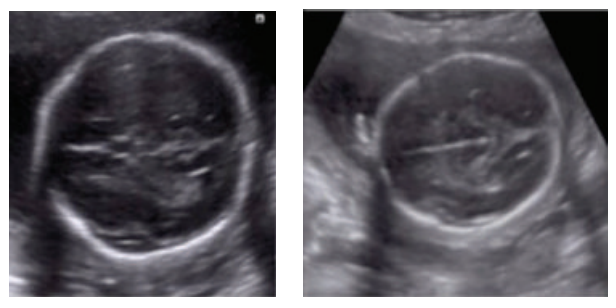

Image D

Measurement of the atrium of the lateral ventricles
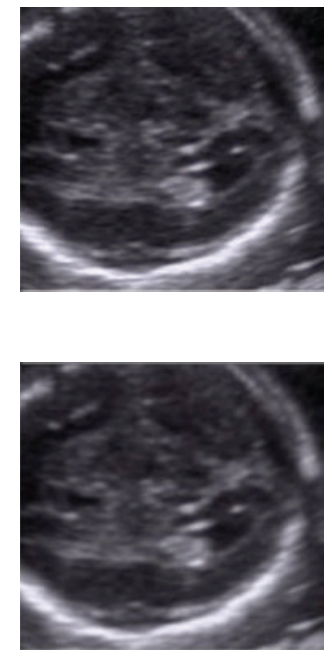

(b)

FIGURE 2: Transventricular, transthalamic, transcerebellar, and measurement of the atrium of the lateral ventricles; (a) original and (b) transmitted image planes of fetal central nervous system.

the most efficient processing signal, and the optimal compression system to generate an image with high diagnostic value [19]. The first studies on the quality of image in teleultrasound examined the transmission with the use of bandwidths such as $128 \mathrm{kbit} / \mathrm{s}, 256 \mathrm{kbit} / \mathrm{s}$, and $384 \mathrm{kbit} / \mathrm{s}$ and suggested that the larger the bandwidth, the greater the amount of information received by the observer, which resulted in a more accurate diagnosis [21-25].

Whereas some researchers have evaluated the quality of transmitted images using analyses made by other radiologists, other researchers have measured image degradation using a careful analysis of the Fourier spectrum and contrast measurements [20]. Some studies have used a robotic arm for real-time transmissions. The results indicate that, although the duration of such tests is longer than that required for an in-person examination, the method could provide diagnostic information that is not available in remote or inaccessible areas [26, 27] (Figure 2).

\section{Advances in Real-Time Transmission}

More recently, a new type of image transmission has emerged under the name of Remote Task Scale, where the expert sees the images transmitted in real time with loss of quality but remotely receives the tests' recorded sequence of images with total quality. This modality can be implemented with the use of broadband technology of medium and high speed and with the use of streaming video $[19,20,28]$.

With the advancement of open networks, image compression technology, and bandwidth levels in many countries, including developing countries, many locales have bandwidth that exceeds the minimum bandwidth recommended for transmission in the initial studies published a decade ago on high-quality synchronous and asynchronous transmissions. Advances in telecommunications and signal processing and the relatively high resolution currently available for portable ultrasound equipment greatly increase the potential clinical applications of teleultrasonography. The remaining technical and logistical barriers include the availability of telecommunications in the desired areas and the training of medical professionals involved in the transmission, reception, and analysis of the images [4, 29].

\section{Training and Education}

According to the World Health Organization (WHO), diagnostic imaging is a necessary procedure for accurately treating at least $25 \%$ of patients worldwide. However, there is currently a lack of imaging services in vast areas of the world, especially in developing countries [1]. Where they do exist, these services are often of poor quality, resulting in misdiagnoses or nondiagnoses [20]. This is because the diagnostic quality of ultrasound images is extremely dependent on the physician and requires a certain level of expertise to obtain all the image planes necessary and interpret their meaning $[11,17]$. Thus, even having the appropriate technology at their disposal, many patients in geographically or socially isolated areas may remain disadvantaged by the lack of a trained professional to operate them [17]. In reality, many small medical centers and isolated locations do not have welltrained sonographers to perform the initial assessments that provide an accurate diagnosis. However, although there have been advances in research in the field of teleultrasonography, studies addressing the training of medical or technical 
personnel are scarce. In our review, only $5 \%$ of the literature on teleultrasonography evaluated education and training of operators. Yet these studies did not systematically quantify the degree to which the professionals involved had improved.

A study in Italy reported that, in relation to the use of teleultrasonography, at least one month of didactic and hands-on training should be performed by operators to ensure that they have acquired sufficient technical competence [30]. Other authors recommended that training should be performed in the workplace of the local doctors by radiologists or sonologists who will evaluate and interpret the images $[10,13,24]$. A more recent study proposed that remote guidance by a specialist be given in real time to instruct inexperienced doctors in acquiring and visualizing the appropriate planes during examinations. The authors considered this educational tool superior to verbal instruction while training doctors at a distance because it enables new skills to be acquired in half the time required using traditional educational practices [15].

Currently, there are no standard training protocols for sonographers in remote locations. To ensure a greater degree of quality control, the images produced locally should be continuously examined via the Internet by competent radiologists and sonologists to ensure that they are following the proper scanning protocols and that the images contain sufficient diagnostic value $[3,4]$. The teleultrasonography and other forms of telemedicine continue to grow; it is important that high quality standards be maintained, or its use may be detrimental to the population. The establishment of a framework to address and examine telemedical errors has been proposed to ensure high levels of quality and safety [31]. Regarding teleultrasonography, gaps in quality include inappropriate scanning protocols, improper scanning technique, inadequate image collection, incomplete examinations, false diagnoses, and nondiagnoses [3, 4, 15].

\section{Clinical Utility}

A significant portion of teleultrasonography research has focused on its use on an outpatient basis with emphasis on the areas of obstetrics and fetal medicine, especially to confirm pregnancy, monitor fetal growth, and evaluate pregnancy related complications such as placenta previa or placenta accreta.

An increasing number of new applications for teleultrasonography have included investigations of almost every organ and system in various medical fields, including the diagnosis of clinical or surgical conditions, examining severely ill patients and guided procedures $[2,15]$. In recent years, many systems have been developed for the paramedic care of trauma patients, both at the scene of an accident and during transport to the hospital, allowing medical teams to adequately prepare to perform required emergency procedures before the patient's arrival [17]. Despite the persistence of barriers in implementing teleultrasonography more broadly and routinely in the initial care administered at the site of trauma, the advances in telecommunications, Internet signal processing, and the high resolution currently available for portable ultrasound equipment increase the scope of applications for teleultrasonography [29]. The remaining technical and logistical barriers are being solved on a daily basis and include improving the availability of telecommunications in desired areas, the reception and analysis of the images, and the training of medical professionals involved in transmission [4].

Several studies on teleultrasonography in remote areas of Australia and Canada have demonstrated its usefulness in gestational and fetal heart examinations [2]. However, most of these studies demonstrated a good correlation between diagnoses made locally and those made remotely [7, 21, 22, 24, 3234]. One example of this correlation was reported in studies conducted in Queensland, Australia. This state, which had a population of 3.3 million, 55\% of whom lived in rural areas, only had two specialized fetal-maternal echography centers, which were located at universities in the capital, Brisbane. The implementation of a teleultrasonography service showed good diagnostic accuracy and identified all of the existing fetal abnormalities in the cases examined. Clinicians for these patients stated that, in the absence of teleultrasonography, they would have sent $1 / 3$ of these patients to the closest specialized ultrasonography center, which was located $1500 \mathrm{~km}$ away $[13,14,35]$.

A similar study was conducted to review the usefulness, accuracy, and benefits of teleultrasonography in primary care hospitals in South Dakota (USA) that care for neonates with suspected congenital heart disease. Two primary hospitals established a telemedicine link with a tertiary center. A pediatric cardiologist interpreted the neonatal teleultrasound images, and the results and recommendations of the specialist were immediately reported to the attending physicians. The study concluded that teleultrasonography could accurately distinguish between neonates who needed immediate cardiac care and those with less critical heart disease, thereby providing immediate diagnoses and appropriate care [36].

\section{Future Directions}

Although it is clear that ultrasonography is an easily adaptable imaging technology, especially for use in developing countries, the mere presence of the equipment does not indicate improvements in patient care [17]. With the continuous development and democratization of the Internet and other information technologies, teleultrasonography has the potential to assist in the provision of specialist services to remote locations. In addition to typical teleultrasonography applications in routine clinical practice, emergency interventional procedures guided by teleultrasonography, such as thoracocentesis, pericardiocentesis, and paracentesis, are already being used experimentally in intensive care units. The routine use of these and other innovations should result in overall improvements in patient care in remote and isolated regions [17].

The future for the application of teleultrasound is potentially enormous, both for the developed countries and for the developing ones. Several efforts have been made in order to increase the simplification of the ultrasound equipment, 


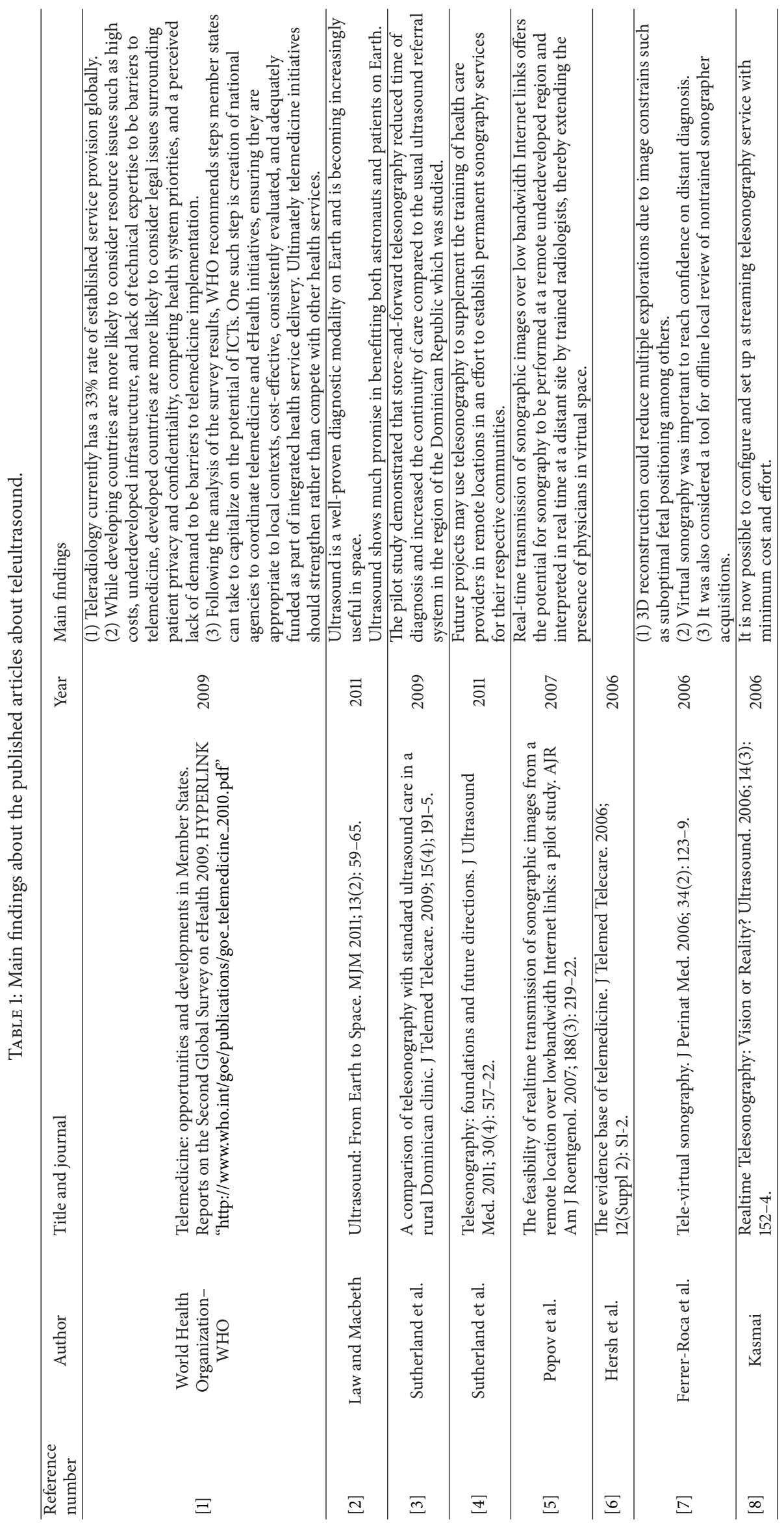




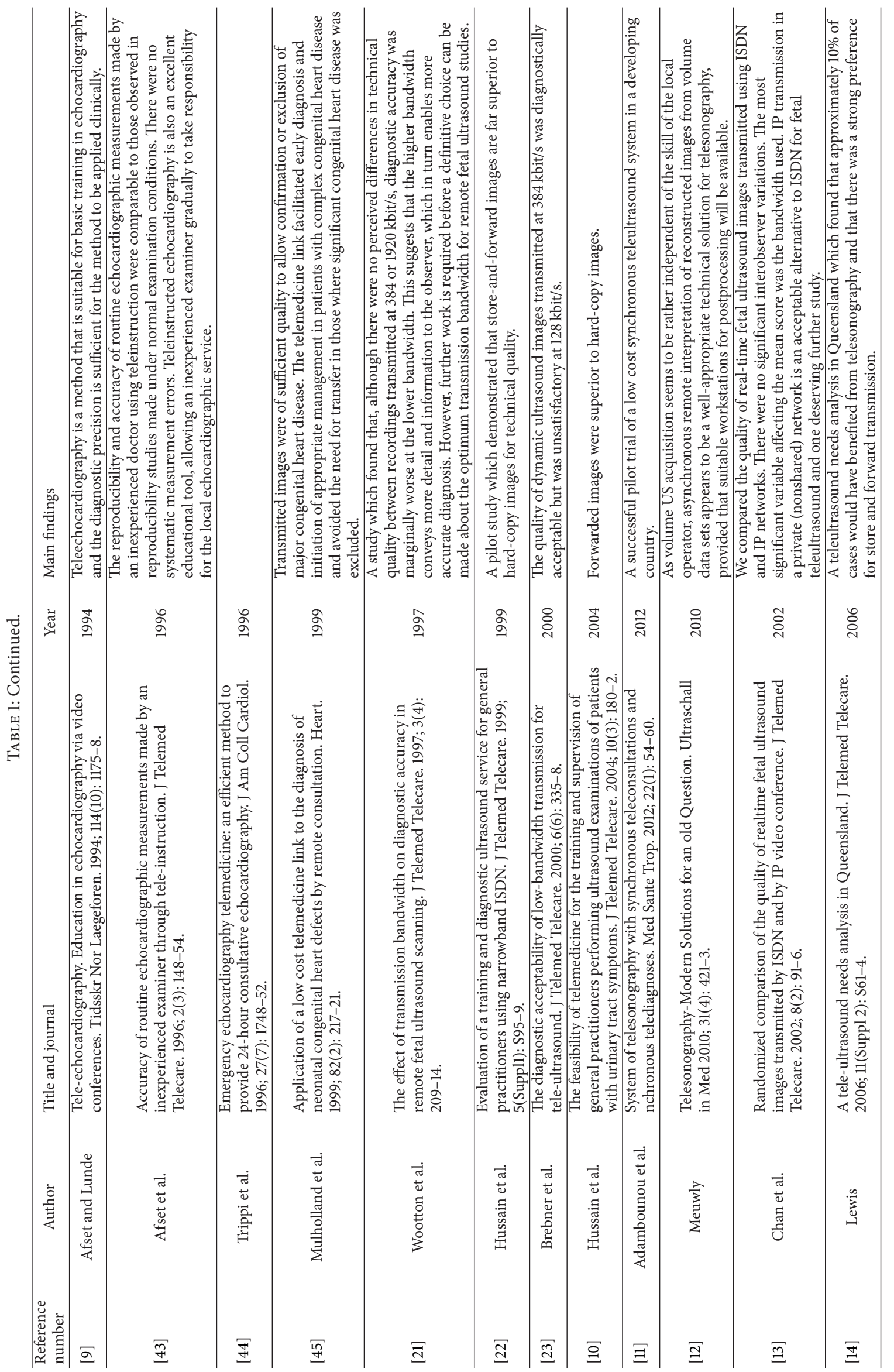




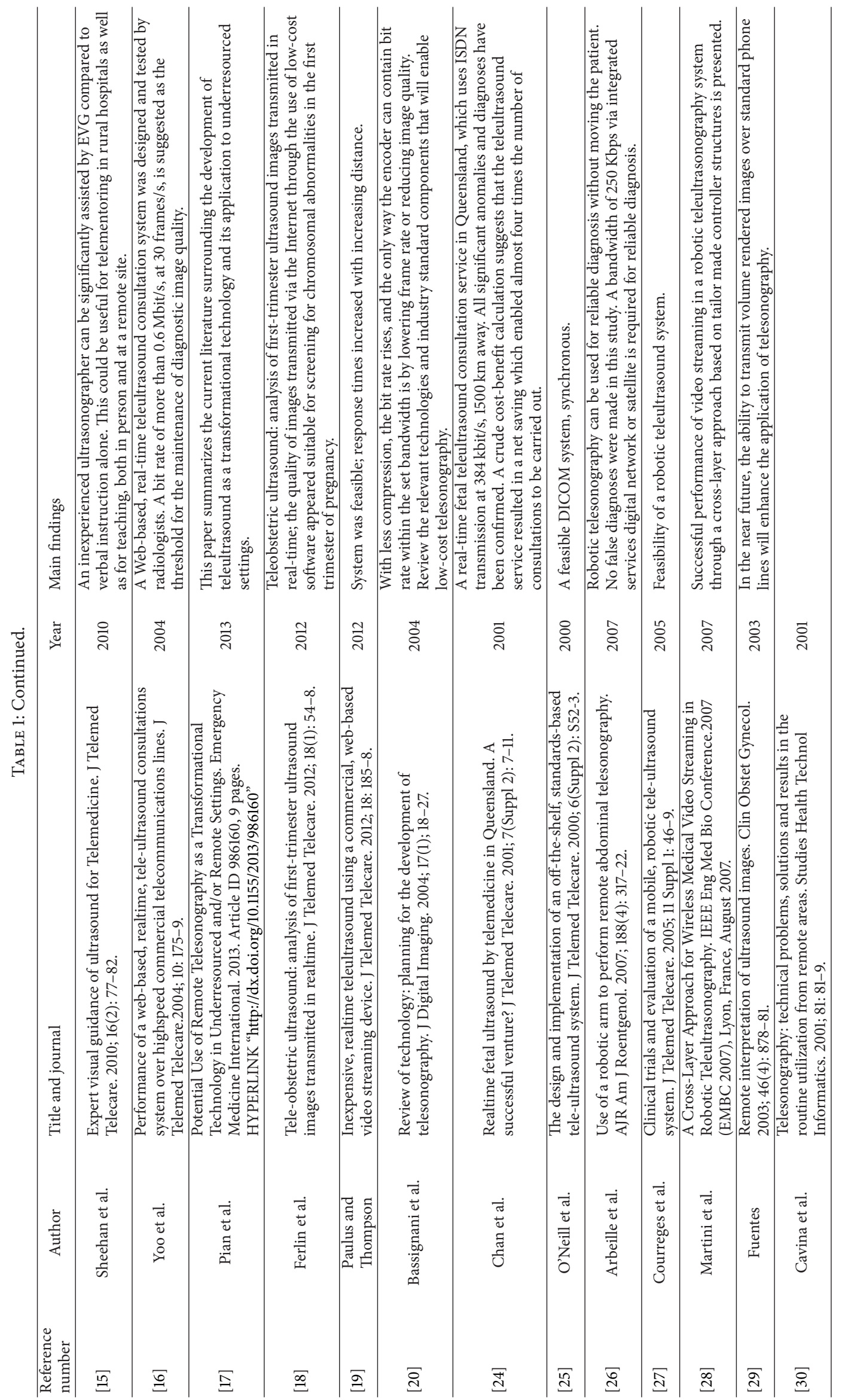




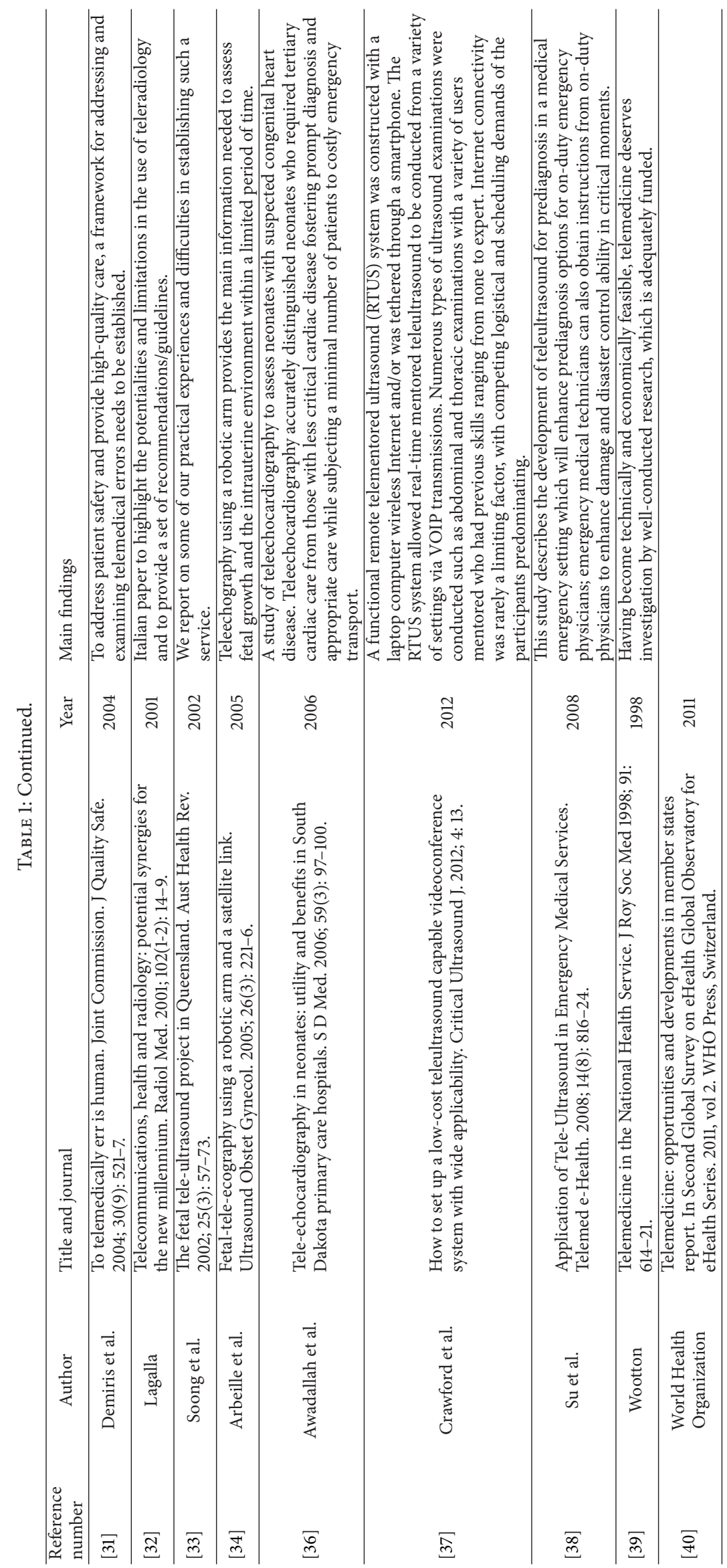


increasing their portability. These advances open new areas of potential applications [37]. In the future, these technologies will be common place and will be first-use technologies, providing rapid and accurate diagnoses and improving the quality of patient care, especially in remote communities [11]. These technologies have led some scholars to consider the tool of teleultrasonography as the "stethoscope of the future" [38].

The routine use of portable ultrasound equipment allied to technological advances may be used for educational purposes in practically any place in the world, providing training of quality and permanent updating for ultrasonography professionals living in remote or needy regions. These innovations combined with educational strategies and the introduction of strict training protocols for health care workers, primarily but not exclusively in remote communities, will allow teleultrasonography to be definitively established as an effective tool in health care and an important tool in educating and training individuals working with ultrasonography. With increased use of ultrasonography, the need for education and training of users becomes clear.

Notably, teleultrasonography has been studied very little for its use in the continuing education of health care providers. Future studies should be conducted not only to quantify the results of patient care but also to measure the degree of training of the professionals involved because performing supervised ultrasound examinations can be used as a tool for a second opinion, encouraging continuous and gradual improvement.

\section{Barriers to the Implementation of Clinical Teleultrasound}

In the 90s, Wooton summarized the critical questions that needed to be approached in order to develop a plan for the implementation of telemedicine: to evaluate the necessary structural changes to incorporate technology, develop an education and training process and formulate guidelines, quality control, and continuous audit [39]. These questions also apply to teleultrasound and are up-to-date even nowadays.

Teleultrasound is a specific type of telemedicine that uses the technological advances for the remote interpretation of ultrasound images. Nevertheless, software, hardware, and/or video-conferences platforms that are necessary for the transmission of images are still highly costly which is one of the main barriers for its implementation in the developing countries [37, 40].

Secondly, although the technological barriers for the development of teleultrasound are continuously disappearing, the nontechnological ones still persist. These barriers include deficits in training and operational protocols, complexity in the use of equipment, and concerns regarding the security and, by extension, the confidentiality of electronically transmitted information $[3,4,41,42]$.

Table 1 summarizes the main findings of published articles about teleultrasound.

\section{Conclusions}

Teleultrasound is a valuable addition to remote medical care for isolated populations with limited access to tertiary healthcare facilities. As some studies point out, the portability and the low cost of the equipment frequently make ultrasonography the only modality of image test available in remote places. The implementation of teleechography in remote or needy places allows for more timely detection of problems, facilitates the obtaining of a second opinion, and reduces the costs of moving. Educational strategies for health agents in remote or needy locations must be continuously researched and developed with the purpose of standardizing training protocols and providing quality assistance to these populations.

\section{Conflict of Interests}

The authors declare that there is no conflict of interests regarding the publication of this paper.

\section{References}

[1] World Health Organization, “Telemedicine: opportunities and developments in Member States. Reports on the Second Global Survey on eHealth," 2009, http://www.who.int/goe/publications/goe_telemedicine_2010.pdf.

[2] J. Law and P. B. Macbeth, "Ultrasound: from earth to space," McGill Journal of Medicine, vol. 13, no. 2, pp. 59-65, 2011.

[3] J. E. Sutherland, H. D. Sutphin, F. Rawlins, K. Redican, and J. Burton, "A comparison of telesonography with standard ultrasound care in a rural Dominican clinic," Journal of Telemedicine and Telecare, vol. 15, no. 4, pp. 191-195, 2009.

[4] J. E. Sutherland, D. Sutphin, K. Redican, and F. Rawlins, "Telesonography: foundations and future directions," Journal of Ultrasound in Medicine, vol. 30, no. 4, pp. 517-522, 2011.

[5] V. Popov, D. Popov, I. Kacar, and R. D. Harris, “The feasibility of real-time transmission of sonographic images from a remote location over low-bandwidth Internet links: a pilot study," The American Journal of Roentgenology, vol. 188, no. 3, pp. W219W222, 2007

[6] W. R. Hersh, D. H. Hickam, and M. Erlichman, "The evidence base of telemedicine: overview of the supplement.," Journal of Telemedicine and Telecare, vol. 12, pp. S1-S2, 2006.

[7] O. Ferrer-Roca, A. Kurjak, J. M. Troyano-Luque, J. B. Arenas, A. L. Mercé, and A. Diaz-Cardama, "Tele-virtual sonography," Journal of Perinatal Medicine, vol. 34, no. 2, pp. 123-129, 2006.

[8] B. Kasmai, "Realtime telesonography: vision or reality?" Ultrasound, vol. 14, no. 3, pp. 152-154, 2006.

[9] J. E. Afset and P. Lunde, "Tele-echocardiography. Education in echocardiography via video conferences," Tidsskrift for den Norske Laegeforening, vol. 114, no. 10, pp. 1175-1178, 1994.

[10] P. Hussain, A. Deshpande, P. Shridhar, G. Saini, and D. Kay, "The feasibility of telemedicine for the training and supervision of general practitioners performing ultrasound examinations of patients with urinary tract symptoms," Journal of Telemedicine and Telecare, vol. 10, no. 3, pp. 180-182, 2004. 
[11] K. Adambounou, F. Farin, A. Boucher et al., "System of telesonography with synchronous teleconsultations and asynchronous telediagnoses," Medecine et Sante Tropicales, vol. 22, no. 1, pp. 54-60, 2012.

[12] J.-Y. Meuwly, “Telesonography—-modern solutions for an old question," Ultraschall in der Medizin, vol. 31, no. 4, pp. 421-423, 2010.

[13] F. Y. Chan, A. Taylor, B. Soong et al., "Randomized comparison of the quality of realtime fetal ultrasound images transmitted by ISDN and by IP video conference," Journal of Telemedicine and Telecare, vol. 8, no. 2, pp. 91-96, 2002.

[14] C. Lewis, "A tele-ultrasound needs analysis in Queensland," Journal of Telemedicine and Telecare, vol. 11, pp. S61-S64, 2006.

[15] F. H. Sheehan, M. A. Ricci, C. Murtagh, H. Clark, and E. L. Bolson, "Expert visual guidance of ultrasound for telemedicine," Journal of Telemedicine and Telecare, vol. 16, no. 2, pp. 77-82, 2010.

[16] S. K. Yoo, D. K. Kim, S. M. Jung, E.-K. Kim, J. S. Lim, and J. H. Kim, "Performance of a Web-based, realtime, teleultrasound consultation system over high-speed commercial telecommunication lines," Journal of Telemedicine and Telecare, vol. 10, no. 3, pp. 175-179, 2004.

[17] L. Pian, L. M. Gillman, P. B. McBeth et al., "Potential use of remote telesonography as a transformational technology in underresourced and/or remote settings," Emergency Medicine International, vol. 2013, Article ID 986160, 9 pages, 2013.

[18] R. M. Ferlin, D. M. Vaz-Oliani, A. C. Ferreira, E. G. Tristão, and A. H. Oliani, "Tele-obstetric ultrasound: analysis of firsttrimester ultrasound images transmitted in realtime," Journal of Telemedicine and Telecare, vol. 18, no. 1, pp. 54-58, 2012.

[19] Y. M. Paulus and N. P. Thompson, "Inexpensive, realtime teleultrasound using a commercial, web-based video streaming device," Journal of Telemedicine and Telecare, vol. 18, no. 4, pp. 185-188, 2012.

[20] M. J. Bassignani, S. J. Dwyer III, J. M. Ciambotti et al., "Review of technology: planning for the development of telesonography," Journal of Digital Imaging, vol. 17, no. 1, pp. 18-27, 2004.

[21] R. Wootton, J. Dornan, N. M. Fisk et al., "The effect of transmission bandwidth on diagnostic accuracy in remote fetal ultrasound scanning," Journal of Telemedicine and Telecare, vol. 3, no. 4, pp. 209-214, 1997.

[22] P. Hussain, D. Melville, R. Mannings, D. Curry, D. Kay, and P. Ford, "Evaluation of a training and diagnostic ultrasound service for general practitioners using narrowband ISDN," Journal of Telemedicine and Telecare, vol. 5, pp. S95-S99, 1999.

[23] J. A. Brebner, H. Ruddick-Bracken, E. M. Brebner et al., "The diagnostic acceptability of low-bandwidth transmission for teleultrasound," Journal of Telemedicine and Telecare, vol. 6, no. 6, pp. 335-338, 2000.

[24] F. Y. Chan, B. Soong, D. Watson, and J. Whitehall, "Realtime fetal ultrasound by telemedicine in Queensland. A successful venture?" Journal of Telemedicine and Telecare, vol. 7, no. 2, pp. S7-S11, 2001.

[25] S. K. O’Neill, D. Allen, and P. D. Brockway, “The design and implementation of an off-the-shelf, standards-based teleultrasound system.", Journal of Telemedicine and Telecare, vol. 6, pp. S52-S53, 2000.

[26] P. Arbeille, A. Capri, J. Ayoub, V. Kieffer, M. Georgescu, and G. Poisson, "Use of a robotic arm to perform remote abdominal telesonography," The American Journal of Roentgenology, vol. 188, no. 4, pp. 317-322, 2007.
[27] F. Courreges, P. Vieyres, R. S. H. Istepanian, P. Arbeille, and C. Bru, "Clinical trials and evaluation of a mobile, robotic teleultrasound system," Journal of Telemedicine and Telecare, vol. 11, no. 1, pp. S46-S49, 2005.

[28] M. G. Martini, R. S. Istepanian, M. Mazzotti, and N. Philip, "A cross-layer approach for wireless medical video streaming in robotic teleultrasonography," in Proceedings of the 29th Annual International Conference of the IEEE Engineering in Medicine and Biology Society (EMBS '07), pp. 3082-3085, Lyon, France, August 2007.

[29] A. Fuentes, "Remote interpretation of ultrasound images," Clinical Obstetrics \& Gynecology, vol. 46, no. 4, pp. 878-881, 2003.

[30] E. Cavina, O. Goletti, P. V. Lippolis, and G. Zocco, Telesonography: Technical Problems, Solutions and Results in the Routine Utilization From Remote Areas, vol. 81 of Studies in Health Technology and Informatics, 2001.

[31] G. Demiris, T. B. Patrick, J. A. Mitchell, and S. E. Waldren, "To telemedically err is human," Joint Commission Journal on Quality and Safety, vol. 30, no. 9, pp. 521-527, 2004.

[32] R. Lagalla, "Telecommunications, health and radiology: potential synergies for the new millennium," Radiologia Medica, vol. 102, no. 1-2, pp. 14-19, 2001.

[33] B. Soong, F. Y. Chan, S. Bloomfield, M. Smith, and D. Watson, "The fetal tele-ultrasound project in Queensland," Australian Health Review, vol. 25, no. 3, pp. 67-73, 2002.

[34] P. Arbeille, J. Ruiz, P. Herve, M. Chevillot, G. Poisson, and F. Perrotin, "Fetal tele-echography using a robotic arm and a satellite link," Ultrasound in Obstetrics and Gynecology, vol. 26, no. 3, pp. 221-226, 2005.

[35] F. Y. Chan, B. Soong, D. Watson, and J. Whitehall, "Realtime fetal ultrasound by telemedicine in Queensland. A successful venture?" Journal of Telemedicine and Telecare, vol. 7, no. 2, pp. 7-11, 2001.

[36] S. Awadallah, I. Halaweish, and F. Kutayli, "Tele-echocardiography in neonates: utility and benefits in South Dakota primary care hospitals," South Dakota Medicine, vol. 59, no. 3, pp. 97100, 2006.

[37] I. Crawford, P. B. McBeth, M. Mitchelson, J. Ferguson, C. Tiruta, and A. W. Kirkpatrick, "How to set up a low cost teleultrasound capable videoconferencing system with wide applicability," Critical Ultrasound Journal, vol. 4, no. 1, p. 13, 2012.

[38] M.-J. Su, H.-M. Ma, C.-I. Ko et al., "Application of teleultrasound in emergency medical services," Telemedicine and $e$ Health, vol. 14, no. 8, pp. 816-824, 2008.

[39] R. Wootton, "Telemedicine in the National Health Service," Journal of the Royal Society of Medicine, vol. 91, no. 12, pp. 614621, 1998.

[40] World Health Organization, Telemedicine: Opportunities and Developments in Member States Report, vol. 2 of Second Global Survey on eHealth Global Observatory for eHealth Series, WHO Press, Geneva, Switzerland, 2011, http://www.who.int/goe/publications/goe_telemedicine_2010.pdf.

[41] J. G. Anderson, "Social, ethical and legal barriers to E-health," International Journal of Medical Informatics, vol. 76, no. 5-6, pp. 480-483, 2007.

[42] K. S. Rheuban, "The role of telemedicine in fostering health-care innovations to address problems of access, specialty shortages and changing patient care needs," Journal of Telemedicine and Telecare, vol. 12, no. 2, pp. S45-S150, 2006. 
[43] J. E. Afset, P. Lunde, and K. Rasmussen, "Accuracy of routine echocardiographic measurements made by an inexperienced examiner through tele-instruction," Journal of Telemedicine and Telecare, vol. 2, no. 3, pp. 148-154, 1996.

[44] J. A. Trippi, K. S. Lee, G. Kopp, D. Nelson, and R. Kovacs, “Emergency echocardiography telemedicine: an efficient method to provide 24-hour consultative echocardiography," Journal of the American College of Cardiology, vol. 27, no. 7, pp. 1748-1752, 1996.

[45] H. C. Mulholland, F. Casey, D. Brown et al., "Application of a low cost telemedicine link to the diagnosis of neonatal congenital heart defects by remote consultation," Heart, vol. 82, no. 2, pp. 217-221, 1999. 

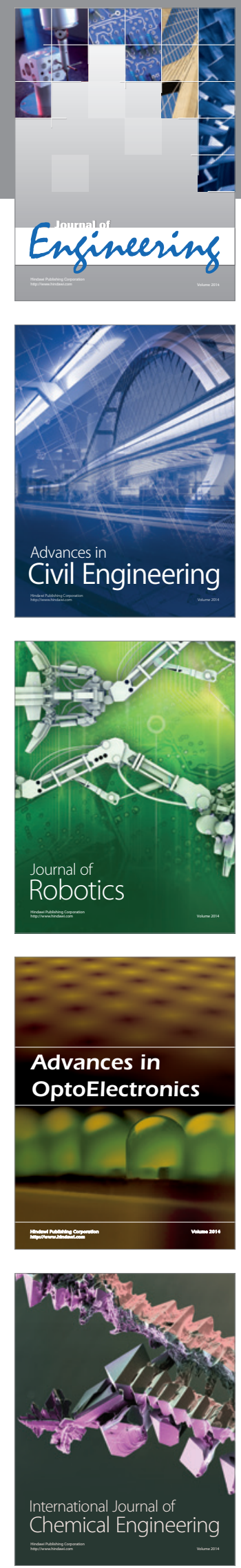

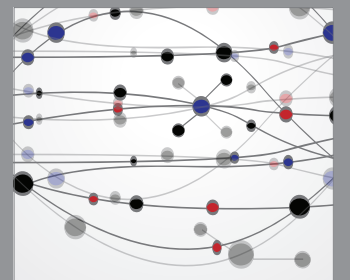

The Scientific World Journal
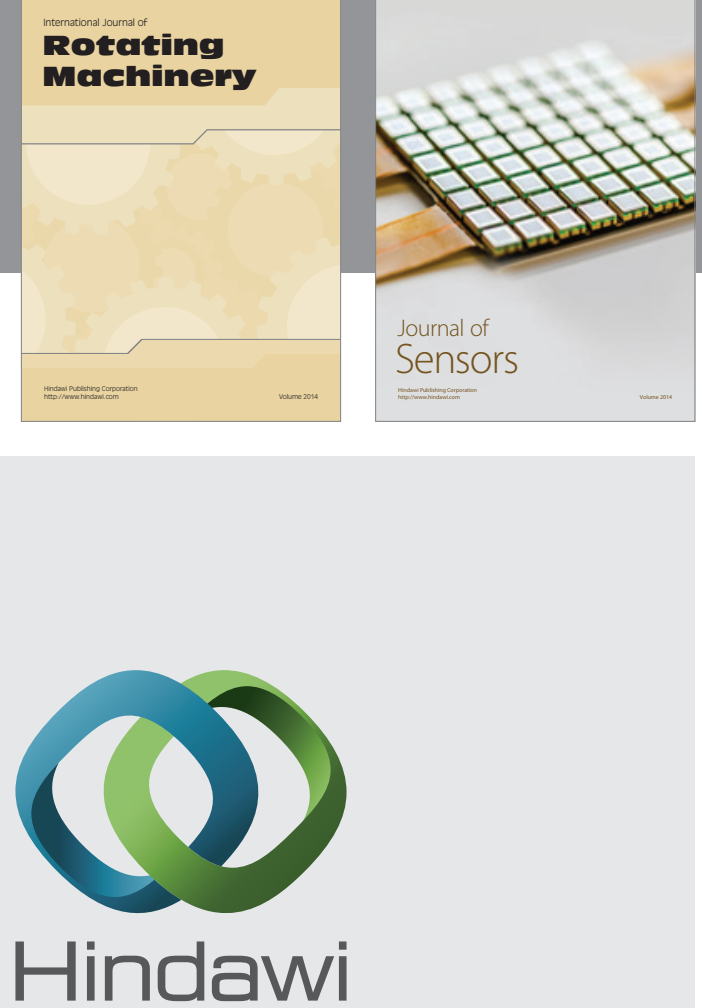

Submit your manuscripts at http://www.hindawi.com
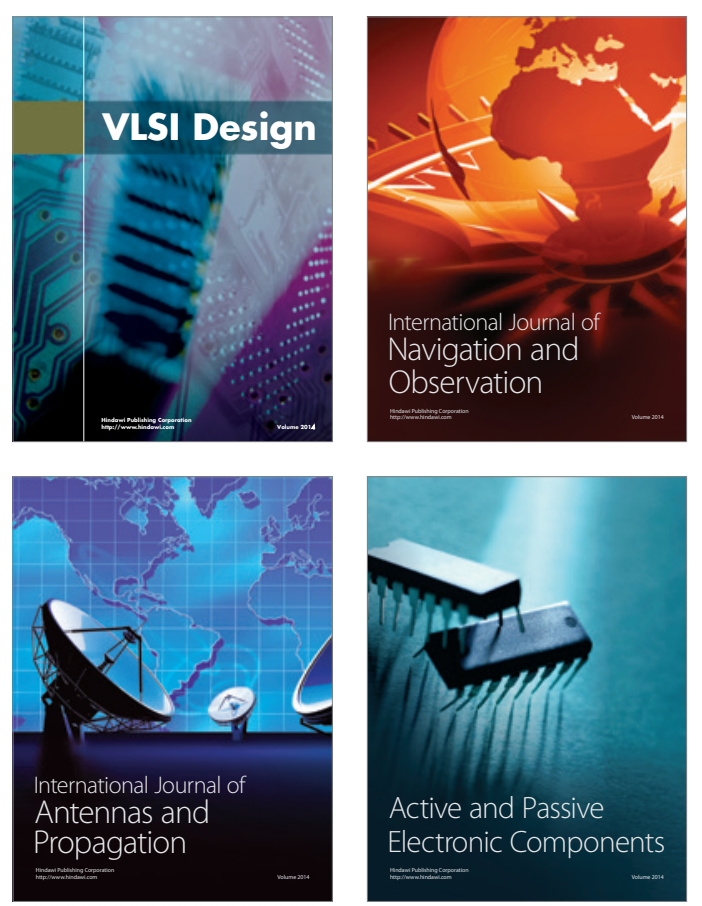
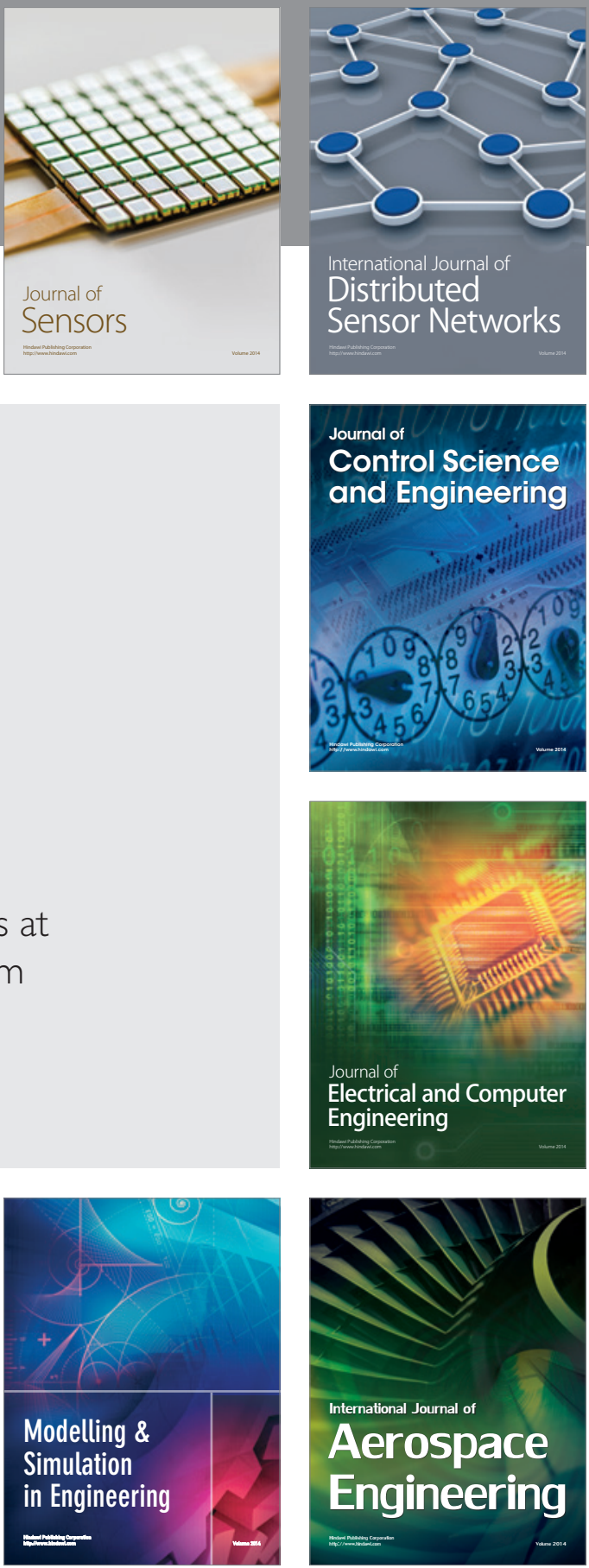

Journal of

Control Science

and Engineering
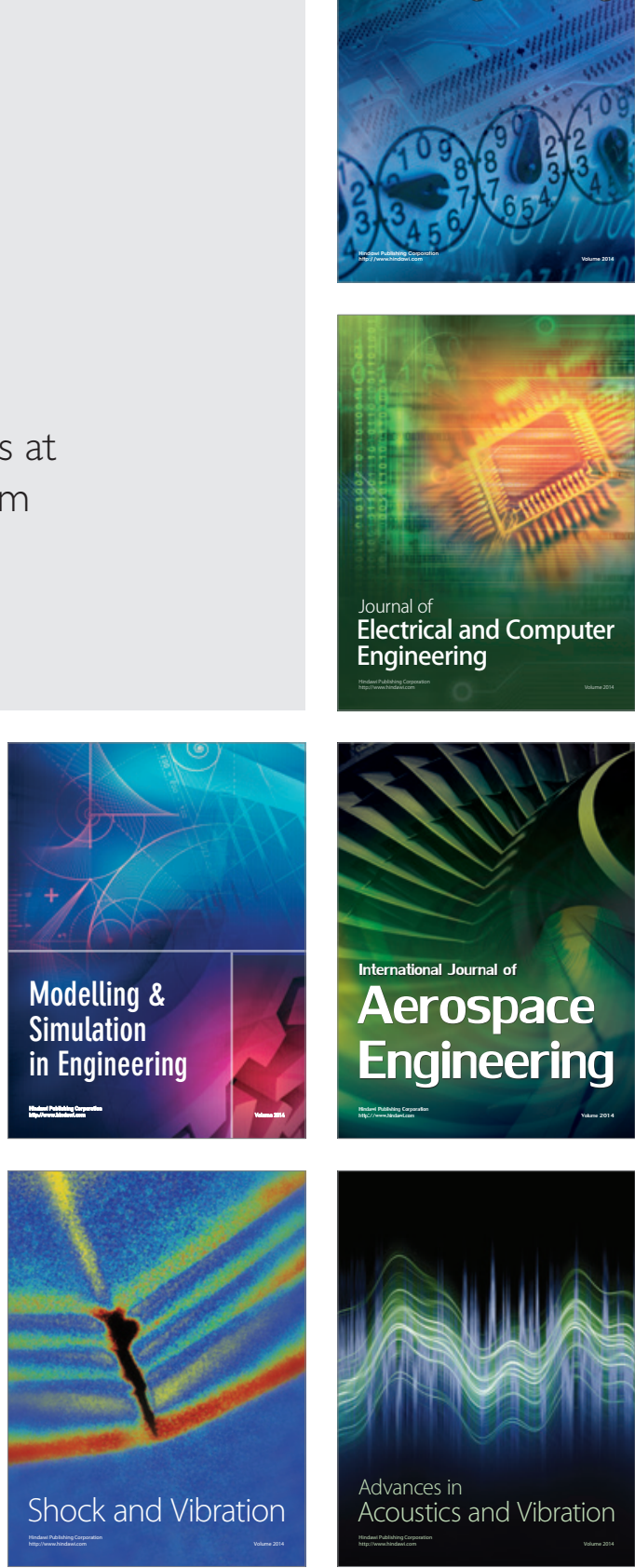